\title{
One-Pot Synthesis of Xanthones and Dixanthones Using Calix[4]arene Sulfonic Acid Under Solvent Free Condition
}

\author{
MOSLEM MANSOUR LAKOURAJ, ${ }^{*}$ HAMED TASHAKKORIAN and MONA ROUHI
}

Department of Organic Chemistry, Faculty of Chemistry, University of Mazanadaran, Babolsar -47416, Iran

Department of Public Health, Faculty of Paramedical, Babol University of Medical Sciences, I. R. Iran

lakouraj@umz.ac.ir

Received 14 Octomber 2012 / Accepted 12 November 2012

\begin{abstract}
An efficient and convenient methodology is introduced for preparation of xanthones and dixanthones through direct coupling of salicylic acids and phenols. It is based on the use of $\mathrm{POCl}_{3}$ and catalytic amount of calix[4]arene sulfonic acid as a highly thermally stable and recyclable organocatalyst. A variety of salicylic acids and phenols were treated with $\mathrm{POCl}_{3}$ and the catalyst in the absence of solvent at $80^{\circ} \mathrm{C}$ which afforded the corresponding xanthones in good to high yields.
\end{abstract}

Keywords: Xanthone, Calix[4]arene Sulfonic Acid, Solvent-free, Catalysis, Ring closure, One-pot synthesis

\section{Introduction}

The xanthones nucleus is the main scaffold of a large category of natural and synthetic materials that demonstrated pharmaceutical properties such as extraordinary anti-inflammatory activity $^{1}$ and act as inhibitors in growth of different human tumor cell lines ${ }^{2}$. Xanthones are known as a common type of antimalarial compounds ${ }^{3}$. In addition, xanthone skeleton displays good thermo-oxidative and hydrolytic stability and cause to have considerable potential as a structural motif in high performance and engineering polymers ${ }^{4}$. Several derivatives of xanthone were isolated from higher plants, fungi and lichens ${ }^{5}$. However; the naturally occurring xanthones are limited to a narrow range of substituents imposed by the biosynthetic pathways. So, in addition to extracting some natural occurring derivatives, several efforts have been employed to synthesize them from their constituent fragments to construct the fused pyranone ring. Therefore a number of methods are developed for the synthesis of new derivatives, including cyclodehydration of 2,2'-dihydroxybenzophenones ${ }^{6}$, electrophilic cycliacylation of 2-aryloxybenzoic acids ${ }^{7}$, tandem coupling-cyclization of arenes with salicylates ${ }^{8}$, oxidative cyclization of 2-phenoxy-benzaldehydes ${ }^{9}$ and nucleophilic aromatic substitution reaction $\left(\mathrm{S}_{\mathrm{N}} \mathrm{Ar}\right)$ for cyclization of $o$-halobenzophenones ${ }^{10}$. 
In cycloacylation-dehydration procedures an excess amount of acid activating agent such as $\mathrm{POCl}_{3}$, PPA and $\mathrm{P}_{2} \mathrm{O}_{5}$ have been applied in the presence of quantitative amount of sulfonic acids or Lewis acids ${ }^{11}$. Unfortunately, these methods suffer from serious drawbacks such as low yield ${ }^{12}$, freshly prepare catalyst ${ }^{13}$ and high temperature $\left(\sim 200{ }^{\circ} \mathrm{C}\right)^{14}$. Along this, to introduce an efficient and convenient method for the synthesis of xanthones and bisxanthones which fulfill the demands for the preparation of new compounds with possibility of having different substituents on the xanthonic nucleus, we explored the convenient procedure using calix[4]arene parasulfonic acid organocatalyst and $\mathrm{POCl}_{3}$ as an acylating agent. The derived products were listed in Table 1 .

\section{Experimental}

The fine chemicals including $p$-tert-butylphenol, formaldehyde solution (37\%), diphenyl ether, ethylacetate, methanol and sodium hydroxide were purchased from Merck (Schuchardt, Germany). Salicylic acid, 2,5-dihydroxy salicylic acid, resorcinol, phosphorus oxychloride and silica gel were obtained from Fluka (Switzerland). Melting points were determined with an Electrothermal 9100 melting point apparatus. IR and ${ }^{1} \mathrm{H}$ NMR spectra were recorded respectively on Bruker FTIR spectrometer and Bruker Avance III $400 \mathrm{MHz}$ NMR spectrometer using tetramethylsilane (TMS) as an internal standard. GC-MS analyses were carried out on a Shimadzu GC 17A gas chromatograph coupled with a MS-QP 5000 Shimadzu mass spectrometer (Tokyo, Japan). Elemental analyses were performed by a CHN Rapid Heraeus elemental analyzer (Wellesley, MA).

\section{General procedures for preparation of xanthones}

To a round bottom flask containing salicylic acid $(1 \mathrm{mmol})$, phenol derivatives $(1 \mathrm{mmol}), \mathrm{POCl}_{3}$ (1.1 mmol) and catalyst $(0.06 \mathrm{mmol})$ was added. The flask was placed in an oil bath and temperature was adjusted to $80{ }^{\circ} \mathrm{C}$. After completion of the reaction which was monitored with TLC, it was poured on to the crushed ice and allowed to stay for an hour. Then extracted with chloroform and washed with saturated aqueous sodium bicarbonate and water and dried. For further purification flash column chromatography was performed using (petroleum ether/ethylacetate, 95:5).

\section{3-Hydroxy xanthone $(\mathbf{1})^{15}$}

The compound 1 was obtained (230.6 mg, 93\%) and characterized according to the described procedure ${ }^{24}$ from compound $1(138 \mathrm{mg}, 1 \mathrm{mmol})$ and resorcinol (110 $\mathrm{mg}, 1$ mmol). (Mp. $\left.179{ }^{\circ} \mathrm{C}\right) \mathrm{IR}(\mathrm{KBr}): 3408,2937,1646,1495,1469,1280,1164,1034 \mathrm{~cm}^{-1}$.

${ }^{1} \mathrm{H} \quad \mathrm{NMR}\left(\mathrm{CDCl}_{3}\right) \delta: 6.64(\mathrm{dd}, \mathrm{J}=7.4 \mathrm{~Hz}, 1 \mathrm{H}), 6.72(\mathrm{~d}, \mathrm{~J}=2.8 \mathrm{~Hz}, 1 \mathrm{H}), 7.28-7.33(\mathrm{~m}$, $1 \mathrm{H}), 7.38(\mathrm{~d}, \mathrm{~J}=8 \mathrm{~Hz}, 1 \mathrm{H}), 7.45(\mathrm{dd}, \mathrm{J}=8.0,1 \mathrm{H}), 7.58-7.63(\mathrm{~m}, 1 \mathrm{H}), 8.04(\mathrm{dd}, \mathrm{J}=8.0,1 \mathrm{H})$, $9.64(\mathrm{~s}, 1 \mathrm{H}) .{ }^{13} \mathrm{C}$ NMR (CDCl3): 101.2, 107.3, 117.8, 121.7, 122.9, 125.2, 127.5, 135.4, 146.2, 155.2, 158.9, 156.8, 177.8; ESI-MS $m / z$ : 212; Anal. Calcd for $\mathrm{C}_{13} \mathrm{H}_{8} \mathrm{O}_{3}: \mathrm{C} 73.58, \mathrm{H}$ 3.76. Found: C, $73.75 ; \mathrm{H}, 3.53$.

\section{2,6-Dihydroxy xanthone $(2)^{16}$}

The compound 2 was synthesized (216.5 $\mathrm{mg}, 82 \%$ ) according to the same procedure mentioned as general procedure, using compound 2 (154 mg, $1 \mathrm{mmol})$ and resorcinol (110 mg, $1 \mathrm{mmol}):$ IR (KBr): 3416, 3010, 2967, 1663, $1457 \mathrm{~cm}^{-1}$.

${ }^{1} \mathrm{H}$ NMR $(\mathrm{CDCl} 3) \delta: 6.47(\mathrm{~d}, \mathrm{~J}=3.2 \mathrm{~Hz}, 1 \mathrm{H}), 6.61(\mathrm{dd}, \mathrm{J}=7.6 \mathrm{~Hz}, 1 \mathrm{H}), 6.78(\mathrm{dd}, \mathrm{J}=7.6,1 \mathrm{H})$, $6.88(\mathrm{~d}, \mathrm{~J}=8 \mathrm{~Hz}, 1 \mathrm{H}), 7.09(\mathrm{~d}, \mathrm{~J}=2.8,1 \mathrm{H}), 7.22(\mathrm{~d}, \mathrm{~J}=7.8,1 \mathrm{H}), 9.49(\mathrm{~s}, 1 \mathrm{H}) 9.51(\mathrm{~s}, 1 \mathrm{H}) .{ }^{13} \mathrm{C}$ NMR $\left(\mathrm{CDCl}_{3}\right): 108.3$, 113.6, 118.2, 120.6, 121.8, 123.4, 127.4, 130.5, 133.4, 136.3, 149.9, 156.5, 177.4. ESI-MS m/z: 232; Anal. Calcd for $\mathrm{C}_{13} \mathrm{H}_{8} \mathrm{O}_{4}$ : C 67.24, H 3.45. Found: C, 67.68; H, 3.73. 


\section{H-Benzo[c]xanthen-7-one $(\mathbf{3})^{8}$}

The compound 3 was obtained in $(253.8 \mathrm{mg}, 90 \%)$ good yield and characterized according to the general procedure mentioned earlier from compound $1(138 \mathrm{mg}, 1 \mathrm{mmol})$ and 1 naphthol (144 mg, $1 \mathrm{mmol})$. IR (KBr): 3061, 1654, 1439, $1153 \mathrm{~cm}^{-1}$.

${ }^{1} \mathrm{H}$ NMR $\left(\mathrm{CDCl}_{3}\right) \delta: 7.36(\mathrm{t}, J=7.8 \mathrm{~Hz}, 1 \mathrm{H}), 7.61-7.7(\mathrm{~m}, 5 \mathrm{H}), 7.78-7.84(\mathrm{~m}, 1 \mathrm{H}), 8.04(\mathrm{~d}$, $J=8.8 \mathrm{~Hz}, 1 \mathrm{H}), 8.25(\mathrm{dd}, J=7.2,1 \mathrm{H}), 8.32(\mathrm{~m}, 1 \mathrm{H}) .{ }^{13} \mathrm{C}$ NMR $\left(\mathrm{CDCl}_{3}\right): 117.5,119.3,121.2$, 122, 123.6, 124.8, 125.3, 125.6, 126.8, 127.5, 128.4, 130.2, 136.4, 137.8, 155.7, 158.6, 179.2. ESI-MS m/z: 234; Anal. Calcd. For $\mathrm{C}_{16} \mathrm{H}_{10} \mathrm{O}_{2}$ : C 82.05, H 4.27. Found: C, 82.41; H, 4.52.

\section{9-Hydroxy-7H-benzo[c]xanthene-7-one $\left(4, \mathrm{C}_{16} \mathrm{H}_{10} \mathrm{O}_{3}\right)$}

The compound 4 was synthesized $(238.4 \mathrm{mg}, 80 \%)$ according to the same procedure mentioned as general procedure, using compound $2(154 \mathrm{mg}, 1 \mathrm{mmol})$ and 1-naphthol (144 mg, $1 \mathrm{mmol}): \mathrm{Mp}>300{ }^{\circ} \mathrm{C}$.

IR (KBr): 3395, 3053, 1664, $1448 \mathrm{~cm}^{-1} .{ }^{1} \mathrm{H}$ NMR $\left(\mathrm{CDCl}_{3}\right) \delta 7.23(\mathrm{~d}, J=7.8 \mathrm{~Hz}, 1 \mathrm{H})$, $7.36(\mathrm{~s}, 1 \mathrm{H}), 7.54-7.62(\mathrm{~m}, 4 \mathrm{H}), 7.85(\mathrm{~d}, J=8.8 \mathrm{~Hz}, 1 \mathrm{H}), 8.03(\mathrm{dd}, J=6.8 \mathrm{~Hz}, 1 \mathrm{H}), 8.25$ $(\mathrm{m}, 1 \mathrm{H}), 9.92(\mathrm{~s}, 1 \mathrm{H}) .{ }^{13} \mathrm{C} \mathrm{NMR}\left(\mathrm{CDCl}_{3}\right) 116.3,116.5,122.4,123.2,123.8,124.6,125.6$, 125.9, 126.8, 127.9, 129.3, 131.5, 135.8, 137.8, 155.4, 156.9, 178.4. ESI-MS m/z: 250; Anal. Calcd for $\mathrm{C}_{16} \mathrm{H}_{10} \mathrm{O}_{3}$ : C 76.8, H 4.01 Found: C, 76.48; H, 4.38.

\section{2,3-Dimethoxyxanthone $(\mathbf{5})^{2}$}

The compound 5 was obtained in $(277.4 \mathrm{mg}, 95 \%)$ good yield and characterized according to the general procedure mentioned earlier from compound 1 (138 $\mathrm{mg}, 1 \mathrm{mmol})$ and 3,4dimethoxyphenol (154 mg, $1 \mathrm{mmol})$. Mp: 167-168 ${ }^{\circ} \mathrm{C}$; IR (KBr) $\mathrm{cm}^{-1}: 3025,1673,1419$, $1244,1138 \mathrm{~cm}^{-1}$.

${ }^{1} \mathrm{H}$ NMR $\left(\mathrm{CDCl}_{3}\right) \delta: 3.79(3 \mathrm{H}, \mathrm{s}, 2-\mathrm{OCH} 3), 4.05(3 \mathrm{H}, \mathrm{s}, 3-\mathrm{OCH} 3), 7.22(1 \mathrm{H}, \mathrm{s}), 7.35$ $(1 \mathrm{H}, \mathrm{m}), 7.41(1 \mathrm{H}, \mathrm{s}), 7.48(1 \mathrm{H}, \mathrm{m}), 7.73(1 \mathrm{H}, \mathrm{m}), 8.10(1 \mathrm{H}, \mathrm{dd}, \mathrm{J}=7.6) ;{ }^{13} \mathrm{C}$ NMR $\left(\mathrm{CDCl}_{3}\right) \delta$ : 56.8, 57.6, 102.4, 106.2, 115.8, 120.7, 122.6, 124.8, 127.2, 136.6, 147.4, 154.1, 157.6, 157.9, 176.8. ESI-MS m/z: 256; Anal. Calcd for $\mathrm{C}_{15} \mathrm{H}_{12} \mathrm{O}_{4}$ : C 70.31, H 4.69 Found: C, 70.06, H 4.94.

\section{7-Hydroxy-2,3-dimethoxyxanthone $\left(6, \mathrm{C}_{15} \mathrm{H}_{12} \mathrm{O}_{5}\right)$}

The compound 6 was synthesized $(268 \mathrm{mg}, 87 \%$ ) according to the same procedure mentioned as general procedure, using compound 2 (154 mg, $1 \mathrm{mmol}$ ) and 3,4-dimethoxy-phenol (154 mg, 1 mmol). Mp: 242-244 C; IR (KBr) cm ${ }^{-1}: 3372,3044,1669,1437,1234,1123 \mathrm{~cm}^{-1}$.

${ }^{1} \mathrm{H}$ NMR $\left(\mathrm{CDCl}_{3}\right) \delta: 3.55(3 \mathrm{H}, \mathrm{s}, 2-\mathrm{OCH} 3), 3.85(3 \mathrm{H}, \mathrm{s}, 3-\mathrm{OCH} 3), 7.04(1 \mathrm{H}, \mathrm{s}), 7.25(1 \mathrm{H}$, $\mathrm{d}, \mathrm{J}=7.8), 7.35(1 \mathrm{H}, \mathrm{s}), 7.53(1 \mathrm{H}, \mathrm{dd}, \mathrm{J}=8.4), 7.84(1 \mathrm{H}, \mathrm{d}, \mathrm{J}=3.6), 9.85(\mathrm{~s}, 1 \mathrm{H}) ;{ }^{13} \mathrm{C} \mathrm{NMR}\left(\mathrm{CDCl}_{3}\right)$

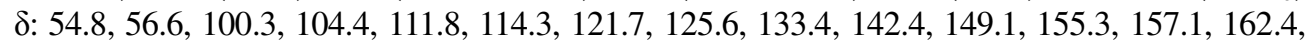
175.2. ESI-MS m/z: 272; Anal. Calcd for $\mathrm{C}_{15} \mathrm{H}_{12} \mathrm{O}_{5}$ : C 66.17, H 4.41 Found: C, 66.43, H 4.12.

\section{2-(2-(9-Oxo-9H-xanthen-2-yl)-propan-2-yl)-9H-xanthene-9-one $\left(7, \mathrm{C}_{29} \mathrm{H}_{20} \mathrm{O}_{4}\right)$}

Using the same method described as general procedure, compound 7 (639.6 mg, 82\%) was obtained with two equivalent $\mathrm{POCl}_{3}$ from compound 1 (276 mg, $\left.2 \mathrm{mmol}\right)$ and bisphenol D (228 mg, $1 \mathrm{mmol}): \mathrm{Mp}>300{ }^{\circ} \mathrm{C} . \mathrm{IR}$ (KBr): 3015, 2947, 1658, $1455 \mathrm{~cm}^{-1}$.

${ }^{1} \mathrm{H}$ NMR (400 MHz, $\left.\mathrm{CDCl}_{3}\right): \delta 1.87(\mathrm{~s}, 6 \mathrm{H}), 6.95(\mathrm{~m}, 2 \mathrm{H}), 7.02(\mathrm{dd}, J=8.4 \mathrm{~Hz}, 2 \mathrm{H}), 7.27$ $(\mathrm{m}, 4 \mathrm{H}), 7.45(\mathrm{~m}, 2 \mathrm{H}), 7.5(\mathrm{~m}, 2 \mathrm{H}), 8.02(\mathrm{dd}, J=8 \mathrm{~Hz}, 2 \mathrm{H}) .{ }^{13} \mathrm{C} \mathrm{NMR}\left(\mathrm{CDCl}_{3}\right) 28.2,55.2$, $109.5,114.5,116.2,117,1,127.3,129.4,130.1,132.2$, 133.9, 135.6, 147.5, 160.3, 170.6. ESIMS m/z: 432, Anal. Calcd for $\mathrm{C}_{29} \mathrm{H}_{20} \mathrm{O}_{4}$ : C 80.55, H 4.63 Found: C, 80.32; H, 4.85 . 


\section{2-(9-Oxo-9H-xanthen-2-ylsulfonyl)-9H-xanthene-9-one $\left(\mathbf{8}, \mathrm{C}_{26} \mathrm{H}_{14} \mathrm{O}_{6} \mathrm{~S}\right)$}

Using the same method described as general procedure, compound $8(577.4 \mathrm{mg}, 72 \%)$ was obtained with two equivalent $\mathrm{POCl}_{3}$ from compound 1 (276 mg, $2 \mathrm{mmol}$ ) and bisphenol $\mathbf{E}$ (250 mg, $1 \mathrm{mmol}$ ): $\mathrm{Mp}>300^{\circ} \mathrm{C}$. IR (KBr): 3028, 2959, 1666, 1445, 1335, $1140 \mathrm{~cm}^{-1}$.

${ }^{1} \mathrm{HNMR}\left(400 \mathrm{MHz}, \mathrm{CDCl}_{3}\right): \delta 7.04(\mathrm{~m}, 2 \mathrm{H}), 7.08(\mathrm{dd}, J=8.4 \mathrm{~Hz}, 2 \mathrm{H}), 7.3(\mathrm{~m}, 4 \mathrm{H})$, $7.53(\mathrm{~m}, 2 \mathrm{H}), 7.59(\mathrm{~m}, 2 \mathrm{H}), 8.1(\mathrm{dd}, J=8 \mathrm{~Hz}, 2 \mathrm{H}) .{ }^{13} \mathrm{C} \mathrm{NMR}\left(\mathrm{CDCl}_{3}\right): 110.4,116.5,118.2$, 119.1, 128.8, 130.5, 132.1, 133.7, 134.8, 139.7, 152.8, 163.3, 169.4. ESI-MS m/z: 454, Anal. Calcd for $\mathrm{C}_{26} \mathrm{H}_{14} \mathrm{O}_{6} \mathrm{~S}$ : C 68.72, H 3.08 Found: C, 68.95; H, 3.41.

\section{2-(1,1,1,3,3,3-Hexafluoro-2-(9-oxo-9H-xanthen-2-yl)propan-2-yl)-9H-xanthene-9-} one $\left(9, \mathrm{C}_{29} \mathrm{H}_{14} \mathrm{O}_{4} \mathrm{~F}_{6}\right)$

Using the same method described as general procedure, compound 9 (621.6 mg, 70\%) was obtained with two equivalent $\mathrm{POCl}_{3}$ from compound 1 (276 mg, $\left.2 \mathrm{mmol}\right)$ and bisphenol $\mathbf{F}$ (336 mg, $1 \mathrm{mmol}$ ): $\mathrm{Mp}>300{ }^{\circ} \mathrm{C}$. IR (KBr): $3038,2963,1668,1452 \mathrm{~cm}^{-1}$.

${ }^{1} \mathrm{H}$ NMR (400 MHz, CDCl 3$): \delta 7.05(\mathrm{~m}, 2 \mathrm{H}), 7.09(\mathrm{dd}, J=8.4 \mathrm{~Hz}, 2 \mathrm{H}), 7.32(\mathrm{~m}, 4 \mathrm{H}), 7.55$ $(\mathrm{m}, 2 \mathrm{H}), 7.6(\mathrm{~m}, 2 \mathrm{H}), 8.15(\mathrm{dd}, J=8 \mathrm{~Hz}, 2 \mathrm{H}) .{ }^{13} \mathrm{C} \mathrm{NMR}\left(\mathrm{CDCl}_{3}\right)$ : 65.7, 111.5, 117.9, 118.7, $119.6,124.2,130.3,131.2,131.7,132.2,134.8,136.8,150.5,162.3,168.4 .{ }^{1} J_{\mathrm{FC}}: 118.7(272 \mathrm{~Hz})$, ESI-MS m/z: 540, Anal. Calcd for $\mathrm{C}_{29} \mathrm{H}_{14} \mathrm{O}_{4} \mathrm{~F}_{6}$ : C 64.44, $\mathrm{H}$ 2.59 Found: C, 64.74; H, 2.76.

\section{2-(9-Oxo-9H-xanthen-2-ylcarbonyl)-9H-xanthene-9-one $\left(\mathbf{1 0}, \mathrm{C}_{27} \mathrm{H}_{14} \mathrm{O}_{5}\right)$}

Using the same method described as general procedure, compound $\mathbf{1 0}(574.5 \mathrm{mg}, 75 \%)$ was obtained with two equivalent $\mathrm{POCl}_{3}$ from compound 1 (276 mg, $\left.2 \mathrm{mmol}\right)$ and bisphenol $\mathbf{G}$ (214 mg, $1 \mathrm{mmol}): \mathrm{Mp}>300{ }^{\circ} \mathrm{C}$.

IR (KBr): 3017, 2953, 1712, 1661, $1464 \mathrm{~cm}^{-1} .{ }^{1} \mathrm{H}$ NMR (400 MHz, $\left.\mathrm{CDCl}_{3}\right): \delta 7.23(\mathrm{~m}, 2 \mathrm{H})$, $7.27(\mathrm{dd}, J=8.4 \mathrm{~Hz}, 2 \mathrm{H}), 7.35(\mathrm{~m}, 4 \mathrm{H}), 7.67(\mathrm{~m}, 2 \mathrm{H}), 7.85(\mathrm{~m}, 2 \mathrm{H}), 8.63(\mathrm{dd}, J=8 \mathrm{~Hz}$, $2 \mathrm{H}) .{ }^{13} \mathrm{C}$ NMR $\left(\mathrm{CDCl}_{3}\right)$ 110.4, 117.2, 118.9, 120.3, 126.8, 130.8, 131.2, 132.7, 133.9, 136.238, 150.2, 161.6, 168.4, 180.4. ESI-MS m/z: 418, Anal. Calcd for $\mathrm{C}_{27} \mathrm{H}_{14} \mathrm{O}_{5}: \mathrm{C} 77.5, \mathrm{H}$ 3.35 Found: C, 77.28; $\mathrm{H}, 3.68$.

\section{2-(9-Oxo-9H-xanthen-2-yl)-9H-xanthene-9-one $\left(11, \mathrm{C}_{26} \mathrm{H}_{14} \mathrm{O}_{4}\right)$}

Using the same method described as general procedure, compound $\mathbf{1 1}(560.9 \mathrm{mg}, 76 \%)$ was obtained with two equivalent $\mathrm{POCl}_{3}$ from compound $\mathbf{1}(276 \mathrm{mg}, 2 \mathrm{mmol})$ and bisphenol $\mathbf{H}$ (186 mg, $1 \mathrm{mmol}): \mathrm{Mp}>300{ }^{\circ} \mathrm{C}$.

IR (KBr): 3010, 2973, 1657, $1448 \mathrm{~cm}^{-1} .{ }^{1} \mathrm{HNMR}\left(400 \mathrm{MHz}, \mathrm{CDCl}_{3}\right): \delta 6.98(\mathrm{~m}, 2 \mathrm{H}), 7.05$ $(\mathrm{dd}, J=8.4 \mathrm{~Hz}, 2 \mathrm{H}), 7.26(\mathrm{~m}, 4 \mathrm{H}), 7.43(\mathrm{~m}, 2 \mathrm{H}), 7.49(\mathrm{~m}, 2 \mathrm{H}), 7.95(\mathrm{dd}, J=8 \mathrm{~Hz}, 2 \mathrm{H}) .{ }^{13} \mathrm{C}$ $\mathrm{NMR}\left(\mathrm{CDCl}_{3}\right)$ : 111.4, 116.5, 117.8, 119.3, 128.2, 130.1, 132.2, 132.7, 133.4, 135.5, 149.2, 158.6, 170.4. ESI-MS m/z: 390, Anal. Calcd for $\mathrm{C}_{26} \mathrm{H}_{14} \mathrm{O}_{4}$ : C 80.1, H 3.59 Found: C, 80.28; H, 3.36.

\section{2-Hydroxy-7-(2-(2-hydroxy-9-oxo-9H-xanthen-7-yl)propan-2-yl)-9H-xanthene-9-one $\left(12, \mathrm{C}_{29} \mathrm{H}_{20} \mathrm{O}_{6}\right)$}

Using the same method described as general procedure, compound $\mathbf{1 2}(641.5 .1 \mathrm{mg}, 76 \%)$ was obtained with two equivalent $\mathrm{POCl}_{3}$ from compound $2(308 \mathrm{mg}, 2 \mathrm{mmol})$ and bisphenol $\mathbf{D}$ (228 mg, $1 \mathrm{mmol}): \mathrm{Mp}>300{ }^{\circ} \mathrm{C}$. IR (KBr): $3342,3020,2957,1648,1445 \mathrm{~cm}^{-1} .{ }^{1} \mathrm{H}$ NMR $(400$ $\left.\mathrm{MHz}_{\mathrm{CDCl}}\right): \delta 1.89(\mathrm{~s}, 6 \mathrm{H}), 6.93(\mathrm{~m}, 2 \mathrm{H}), 6.98(\mathrm{dd}, J=8.0 \mathrm{~Hz}, 2 \mathrm{H}), 7.10(\mathrm{~m}, 2 \mathrm{H}), 7.34$ $(\mathrm{m}, 2 \mathrm{H}), 7.39(\mathrm{~m}, 2 \mathrm{H}), 7.85(\mathrm{dd}, J=7.6 \mathrm{~Hz}, 2 \mathrm{H}) .9 .65(\mathrm{~s}, 2 \mathrm{H}) .{ }^{13} \mathrm{C} \mathrm{NMR}\left(\mathrm{CDCl}_{3}\right): 29.5,63.2$, $110.5,113.5,118.1,119.9,124.3,128.3,130.5,132.6,133.3,135.4,146.8,161.3,169.7$. ESIMS m/z: 464, Anal. Calcd. for $\mathrm{C}_{29} \mathrm{H}_{20} \mathrm{O}_{6}$ : C 75.1, H 4.31 Found: C, 75.37; H, 4.69. 
2-(2-Hydroxy-9-oxo-9H-xanthen-7-ylsulfonyl)-7-hydroxy-9H-xanthene-9-one $\left(13, \mathrm{C}_{26} \mathrm{H}_{14} \mathrm{O}_{8} \mathrm{~S}\right)$

Using the same method described as general procedure, compound $13(562.9 \mathrm{mg}, 65 \%)$ was obtained with two equivalent $\mathrm{POCl}_{3}$ from compound 2 (308 $\left.\mathrm{mg}, 2 \mathrm{mmol}\right)$ and bisphenol $\mathbf{E}$ (250 mg, $1 \mathrm{mmol}$ ): $\mathrm{Mp}>300{ }^{\circ} \mathrm{C}$. IR (KBr): 3295, 3025, 2948, 1660, 1454, 1343, $1132 \mathrm{~cm}^{-1}$.

${ }^{1} \mathrm{H}$ NMR $\left(400 \mathrm{MHz}, \mathrm{CDCl}_{3}\right): \delta 6.99(\mathrm{~m}, 2 \mathrm{H}), 7.02(\mathrm{dd}, J=8.0 \mathrm{~Hz}, 2 \mathrm{H}), 7.10(\mathrm{~m}, 2 \mathrm{H})$, $7.46(\mathrm{~m}, 2 \mathrm{H}), 7.54(\mathrm{~m}, 2 \mathrm{H}), 7.95(\mathrm{dd}, J=7.6 \mathrm{~Hz}, 2 \mathrm{H}) 9.72(\mathrm{~s}, 2 \mathrm{H}) .{ }^{13} \mathrm{C}$ NMR $\left(\mathrm{CDCl}_{3}\right)$ : $110.8,112.8,118.6,120,5,125.3,128.8,131.6,133.7,134.2,137.8,147.1,163.5,171.4$. ESI-MS m/z: 486, Anal. Calcd for $\mathrm{C}_{26} \mathrm{H}_{14} \mathrm{O}_{8} \mathrm{~S}$ : C 64.2, H 2.88 Found: C, 64.48; H, 2.54.

2-(1,1,1,3,3,3-Hexafluoro-2-(2-hydroxy-9-oxo-9H-xanthen-7-yl)propan-2-yl)-7-hydroxy -9H-xanthene-9-one $\left(14, \mathrm{C}_{29} \mathrm{H}_{14} \mathrm{O}_{6} \mathrm{~F}_{6}\right)$

Using the same method described as general procedure, compound $14(618.8 \mathrm{mg}, 65 \%)$ was obtained with two equivalent $\mathrm{POCl}_{3}$ from compound 2 (308 $\left.\mathrm{mg}, 2 \mathrm{mmol}\right)$ and bisphenol $\mathbf{F}$ (336 mg, 1mmol): $\mathrm{Mp}>300{ }^{\circ} \mathrm{C}$. IR (KBr): 3342, 3048, 2976, 1670, $1447 \mathrm{~cm}^{-1}$.

${ }^{1} \mathrm{H}$ NMR $\left(400 \mathrm{MHz}, \mathrm{CDCl}_{3}\right): \delta 6.99(\mathrm{~m}, 2 \mathrm{H}), 7.04(\mathrm{dd}, J=8 \mathrm{~Hz}, 2 \mathrm{H}), 7.19(\mathrm{~m}, 2 \mathrm{H})$, $7.49(\mathrm{~m}, 2 \mathrm{H}), 7.54(\mathrm{~m}, 2 \mathrm{H}), 8.04(\mathrm{dd}, J=7.6 \mathrm{~Hz}, 2 \mathrm{H}) 9.83(\mathrm{~s}, 2 \mathrm{H}) .{ }^{13} \mathrm{C} \mathrm{NMR}\left(\mathrm{CDCl}_{3}\right): 66.9$, 106.5, 110.9, 113.6, 115.3, 120.7, 122.1, 129.4, 130.5, 131.1, 132.4, 133.7, 150.8, 161.7, 168.4. ${ }^{1} J_{\mathrm{FC}}$ : $120.7(264 \mathrm{~Hz})$. ESI-MS $m / z$ : 572, Anal. Calcd for $\mathrm{C}_{29} \mathrm{H}_{14} \mathrm{O}_{6} \mathrm{~F}_{6}: \mathrm{C} 60.83, \mathrm{H} 2.45$ Found: C, 60.48; H, 2.71.

2-(2-Hydroxy-9-oxo-9H-xanthen-7-ylcarbonyl)-7-hydroxy-9H-xanthene-9-one $\left(15, \mathrm{C}_{27} \mathrm{H}_{14} \mathrm{O}_{7}\right)$

Using the same method described as general procedure, compound 15 (564.4 mg, 68\%) was obtained with two equivalent $\mathrm{POCl}_{3}$ from compound 2 (308 $\left.\mathrm{mg}, 2 \mathrm{mmol}\right)$ and bisphenol $\mathbf{G}$ (214 mg, $1 \mathrm{mmol}): \mathrm{Mp}>300{ }^{\circ} \mathrm{C}$. IR (KBr): 3310, 3022, 2965, 1714, 1657, $1451 \mathrm{~cm}^{-1}$.

${ }^{1} \mathrm{H}$ NMR (400 MHz, $\left.\mathrm{CDCl}_{3}\right): \delta 7.17(\mathrm{~m}, 2 \mathrm{H}), 7.21(\mathrm{dd}, J=8 \mathrm{~Hz}, 2 \mathrm{H}), 7.27(\mathrm{~m}, 2 \mathrm{H}), 7.40$ $(\mathrm{m}, 2 \mathrm{H}), 7.70(\mathrm{~m}, 2 \mathrm{H}), 8.35(\mathrm{dd}, J=7.6 \mathrm{~Hz}, 2 \mathrm{H}), 9.81(\mathrm{~s}, 2 \mathrm{H}) .{ }^{13} \mathrm{C} \mathrm{NMR}\left(\mathrm{CDCl}_{3}\right)$ : 106.8, 110.2, 111.8, 114,7, 127.6, 128.1, 129.5, 130.2, 131.6, 137.8, 150.7, 159.8, 169.8, 179.6. ESI-MS $m / z$ : 450, Anal. Calcd for $\mathrm{C}_{27} \mathrm{H}_{14} \mathrm{O}_{7}$ : C 72.01, H, 3.11 Found: C, 72.27; H, 3.42.

\section{2-Hydroxy-7-(2-hydroxy-9-oxo-9H-xanthen-7-yl)-9H-xanthene-9-one $\left(\mathbf{1 6}, \mathrm{C}_{26} \mathrm{H}_{14} \mathrm{O}_{6}\right)$}

Using the same method described as general procedure, compound $\mathbf{1 0}(577.5 \mathrm{mg}, 72 \%)$ was obtained with two equivalent $\mathrm{POCl}_{3}$ from compound 2 (308 mg, $\left.2 \mathrm{mmol}\right)$ and bisphenol $\mathbf{H}$ (186 mg, $1 \mathrm{mmol}$ ): $\mathrm{Mp}>300{ }^{\circ} \mathrm{C}$. IR (KBr): $3304,3028,2970,1667,1446 \mathrm{~cm}^{-1}$.

${ }^{1} \mathrm{H}$ NMR $\left(400 \mathrm{MHz}, \mathrm{CDCl}_{3}\right): \delta 6.95(\mathrm{~m}, 2 \mathrm{H}), 7.01(\mathrm{dd}, J=8 \mathrm{~Hz}, 2 \mathrm{H}), 7.11(\mathrm{~m}, 2 \mathrm{H})$, $7.41(\mathrm{~m}, 2 \mathrm{H}), 7.45(\mathrm{~m}, 2 \mathrm{H}), 7.95(\mathrm{dd}, J=7.6 \mathrm{~Hz}, 2 \mathrm{H}) 9.67(\mathrm{~s}, 2 \mathrm{H}) .{ }^{13} \mathrm{C}$ NMR $\left(\mathrm{CDCl}_{3}\right)$ : 107.2, 109.8, 110.4, 113,6, 128.5, 129.2, 129.9, 130.8, 131.3, 137.4, 149.8, 158.6, 169.8. ESI-MS m/z: 422, Anal. Calcd. for $\mathrm{C}_{26} \mathrm{H}_{14} \mathrm{O}_{6}$ : C 73.9, H, 3.31 Found: C, 73.52; H, 3.69.

\section{Results and Discussion}

Calixarenes as macrocyclic compounds bearing different kinds of functional groups which form complex with different transition metals have been reported as desirable ligands in organometallic catalysis ${ }^{17}$. Calix[4]arene tetrasulfonic acid has shown some interesting capabilities in preparation of conducting polymer, polyaniline, as a doping agent ${ }^{18}$, incapsulating to Topotecan for improving solubility in chemotherapy ${ }^{19}$ and proved to be 
suitable for bio-pharmaceutical applications ${ }^{20}$. More recently, the role of calixarene sulfonic acid in Mannich type reaction ${ }^{21}$, allylic alkylation reactions ${ }^{22}$ and esterification ${ }^{23}$ has been disclosed as a nontoxic and reusable organocatalyst.

In continuation of our recent works on the use of recyclable catalyst in organic transformations ${ }^{24}$ and to introduce a general methodology for the preparation of bishydroxy xanthones as a precursor for polyxanthones and promising building motif for the new type of potential $\alpha$-glucosidase inhibitors ${ }^{13}$, herein we wish to report a highly efficient and general procedure for the one-pot construction of xanthone derivatives based on direct cyclization through acylation-dehydration of salicylic acids and phenols under solvent free condition using calix[4]arene sulfonic acid organocatalyst and $\mathrm{POCl}_{3}$. For this purpose, parent calixarene was synthesized according to Gutsche et al. procedure published in literature using $p$-tertbutylphenol and formaldehyde ${ }^{25}$. Then, it was detertiobutylated and sulfonated simultaneously using concentrated sulfuric acid using Shinkai method (Scheme 1) ${ }^{26}$. The completion of the sulfonation was determined by controlling the complete dissolution of the aliquots in water. After purification according to the procedure described in the literature, the obtained product was used as an efficient acidic organocatalysis.

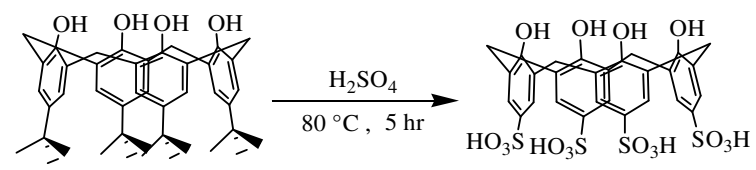

Scheme 1. Sulfonation of calix[4]arne using Shinkai method

To explore the potential activity of sulfonic acid functionalized calixarene, preparation of xanthone derivatives with salicylic acids and a variety of phenols were studied using catalytic amount of $(0.06 \mathrm{mmol})$ organocatalyst (Scheme 2$)$.

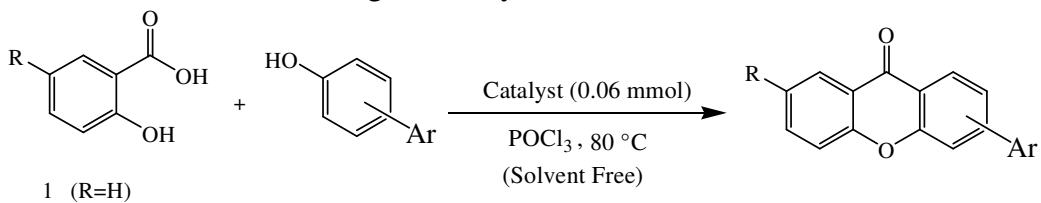

$$
2 \stackrel{(\mathrm{R}=\mathrm{H})}{(\mathrm{OH})}
$$<smiles>Oc1cccc(O)c1</smiles>

(A)

(B)

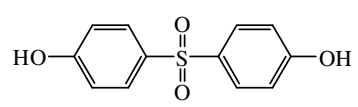

(E)

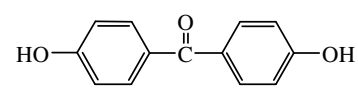

(G)<smiles>COc1ccc(O)cc1OC</smiles>

(D)

(C)

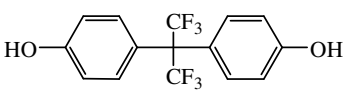

(F)

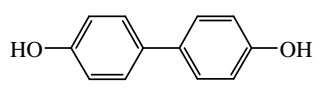

(H)

Scheme 2. Preparation of xanthone derivatives with salicylic acid and a variety of phenols using calix[4]arene sulfonic acid

The results (Table 1) clearly indicated good to high yields for this transformation by using an equimolar amount (which is important from environmental point of view) of phosphorus 
oxychloride as an acylating agent. This procedure demonstrates direct condensation of different salicylic acids and phenols via acylation followed by in situ cyclization of dihydroxy benzophenone intermediates. The results and overall yields indicate that some functional groups accelerate the ring closure and promote the yields obviously.

Table 1. Xanthones and dixanthones prepared from tetrasulfonic acid calixarene

Entry Substrate




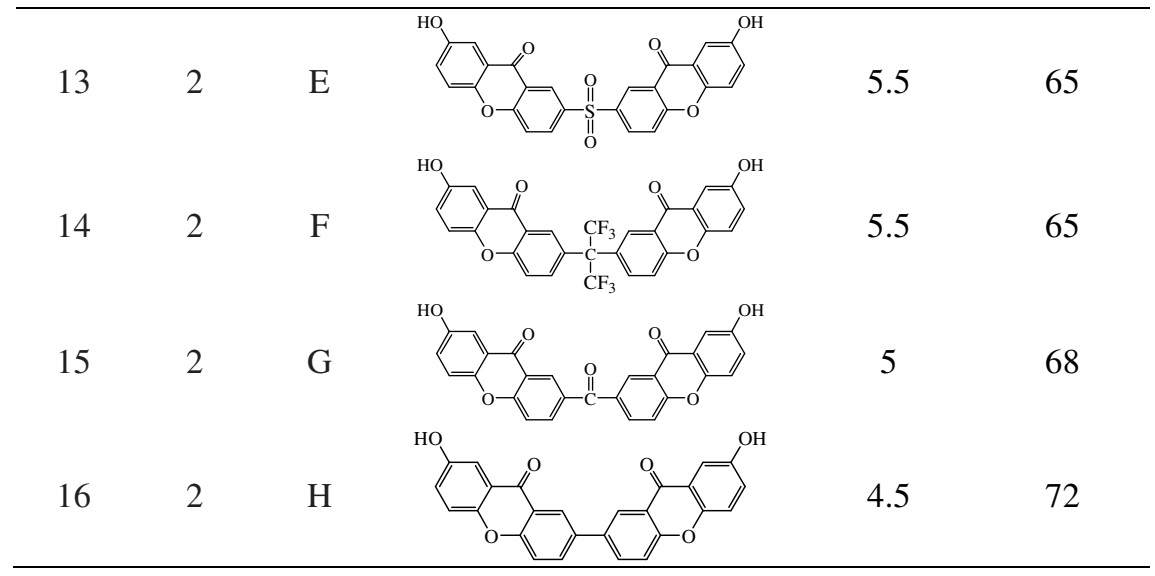

As can be seen in Table 1, dimethoxy phenol produced the corresponding Xanthones in high yield, while phenols bearing sulfonyl and carbonyl groups showed lower reactivity and the corresponding xanthones were obtained in moderate yields. Products formation from 5-hydroxysalisylic acid took more time and relatively lower yields were afforded. On the other hand, treatment of 5-hydroxysalicylic acid with different bisphenols gave novel dixanthonic compounds which would be applicable in preparation of high-performance polymers. The polyester and polyether formation of these monomers are currently under investigation in our laboratory.

For industrial concern and notability of our work especially with these promising polymerizable bishydroxy xanthones (entries 12-16), reusability of the catalyst has a great importance. Therefore, after completion of each reaction, catalyst was simply recovered from the reaction mixture by treating the precipitate with deionized water to dissolve the catalyst. Finally, on filtration, water was evaporated and the organocatalyst dried and reused in successive reactions. The recycled catalyst was found to be highly efficient even after four cycles of use (Figure 1).

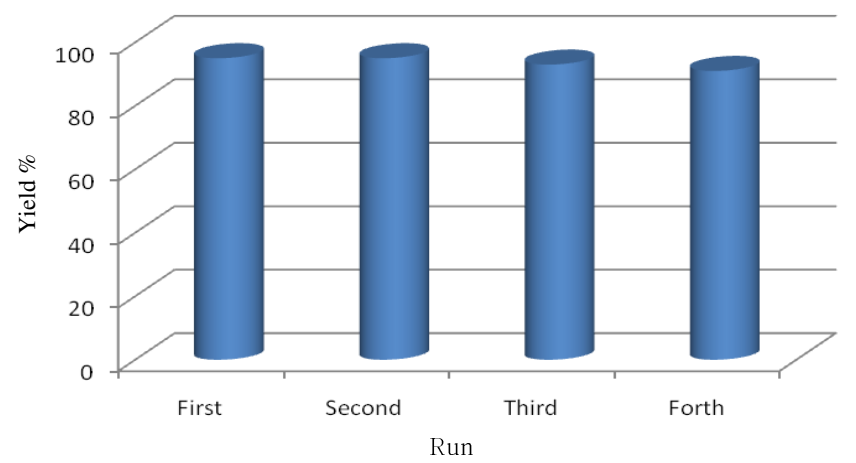

Figure 1. Efficiency of recycled calixarene sulfonic acid in the synthesis of xanthones

\section{Conclusion}

In conclusion, an one pot and applicable procedure for preparation of novel xanthones and dixanthones using efficient and reusable organocatalyst known as $p$-sulfonic acid calix[4]arene has suggested. Herein, we employed this valuable catalyst along with acylating agent for annulations of xanthonic ring under solvent free condition. This study can attracts 
high attention of the researchers who looking for environmentally friendly procedures based on less harmful catalysts such as metal free catalysts or recyclable ones which are more desirable especially in pharmaceutical and food industries. Moreover, promoting the chemical transformation with appreciable conversion of the substrates to synthesize interesting bisxanthone monomers with attractive capabilities was accomplished.

\section{Acknowledgment}

The financial supports by Research Council of University of Mazandaran are gratefully acknowledged.

\section{References}

1. (a) Lin C N, Chung M I, Liou S J, Lee T H and Wang J P, J Pharm Pharmacol., 1996, 48(5), 532-538; (b) Schwaebe M K, Moran T J and Whitten J P, Tetrahedron Lett., 2005, 46, 827-829; (c) Librowski T, Czarnecki R, Czekaj T and Marona H, Medicina, 2005, 41(1), 54-58.

2. Madalena P, Fatima C, Maria E S, Maria S J Nascimentoa and Madalena P, Bioorg Med Chem., 2002, 10, 3725-3730.

3. Ignatushchenko M V, Winter R W, Bachinger H P, Hinrichs D J and Riscoe M K, FEBS Lett., 1997, 409(1), 67-73.

4. Colquhoun H M, Lewis D F and Williams D J, Org Lett., 2001, 3(15), 2337-2340.

5. Cardona M L, Fernandez M I, Pedro J R and Serrano A, Phytochemistry, 1990, 29(9), 3003-3006.

6. Bichan D J and Yates P, Can J Chem., 1975, 53, 2054-2063.

7. Olah G A, Mathew T, Farnia M and Prakash G K S, Synlett, 1999, 1067-1068 and references cited therein.

8. Zhao J and Larock R C, J Org Chem., 2007, 72(2), 583-588.

9. Okogun J I and Okwute K S, J Chem Soc., Chem Commun., 1975, 8-9. DOI: $10.1039 / \mathrm{C} 39750000008$

10. Hintermann L, Masuo R and Suzuki K, Org Lett., 2008, 10(21), 4859-4862.

11. (a) Varache-Lembège $M$, Moreau S, Larrouture S, Montaudon D, Robert J and Nuhrich A, Eur J Med Chem., 2008, 43(6), 1336-1343; (b) Fonteneau N, Martin P, Mondon M, Ficheux H and Gesson J P, Tetrahedron, 2001, 57(44), 9131-9135.

12. Liu Y, Zou L, Ma L, Chen W H, Wang B and Xu Z L, Bioorg Med Chem., 2006, 14(16), 5683-5690.

13. Liu Y, Ma L, Chen W H, Wang B and Xu Z L, Bioorg Med Chem., 2007, 15(8), 2810-2814.

14. Sousa E, Paiva A, Nazareth, N, Gales, L, Damas, A M, Nascimento M S J and Pinto M, Euro J Med Chem., 2009, 44(9), 3830-3835.

15. Gnerre C, Thull U, Gaillar P, Carrupt P A, Testa B, Fernandes E, Silva F, Pinto M, Pinto M M M, Wolfender J L, Hostettman K and Cruciani G, Helv Chim Acta, 2001, 84(3), 552-570.

16. Fatel G F and Trivedi K N, Synth Commun., 1989, 19(9-10), 1641-1647.

17. Notestein J M, Iglesia E and Katz A, J Am Chem Soc., 2004, 126, 16478-16486; (b) Redshaw C, Clowes L, Hughes D L, Elsegood M R J and Yamato T, Organometallics, 2011, 30(21), 5620-5624; (c) Homden D M and Redshaw C, Chem Rev., 2008, 108(12), 5086-5130.

18. Davey J M, Too C O, Ralph S F, Kane-Maguire L A P, Wallace G G and Ashton C. Partridge, Macromol., 2000, 33(19), 7044-7050. 
19. Wang G S, Zhang H Y, Ding F and Liu Y, J Incl Phenom Macrocycl Chem., 2011, 69, 85.

20. Castanheiro R A P, Silva A M S, Campos N A N, Nascimento M S J and Pinto M M M, Pharmaceuticals, 2009, 2(2), 33-43.

21. da Silva D L, Fernandes S A, Sabino A A and de Fatima A, Tetrahedron Lett., 2011, 52(48), 6328-6330; (b) Shimizu S, Shimada N and Sasaki Y, Green Chem., 2006, 8(7), 608-614.

22. Liu L, Wang Y L, Han Y C and Chen Y J, Green Chem., 2008, 10(6), 635-640.

23. Fernandes S A, Natalino R, Gazolla P A R, da Silva M J and Jham G N, Tetrahedron Lett., 2012, 53(13), 1630-1633.

24. Lakouraj M M, Tajbakhsh M and Tashakkorian H, Lett Org Chem., 2007, 4, 75-79; (b) Lakouraj M M, Tajbakhsh M and Tashakkorian H, Monatsh für Chem., 2007, 138, 83.

25. Gutsche C D, Calixarenes revisited, Stoddart J F, (Ed)., The Royal Society of Chemistry: Cambridge, 1998 and references cited therein.

26. Shinkai S, Araki K, Tsubaki T, Arimura T and Manabe O, J Chem Soc Perkin Trans., 1987, 2297. 